\title{
Fast Growth of Sulfuric Acid Aerosol Activated by External Factors for Enhanced Emission Control
}

\author{
Zhengda Yang ${ }^{1,2}$, Chenghang Zheng ${ }^{1}$, Qingyi $\mathrm{Li}^{3}$, Hao Zheng ${ }^{1}$, Haitao Zhao ${ }^{1}$, Xiang Gao ${ }^{1 *}$ \\ 1. State Key Lab of Clean Energy Utilization, Zhejiang University, Hangzhou 310027, P. R. China \\ 2. College of New Energy, China University of Petroleum (East China), Qingdao 266580, P. R. China \\ 3. Zhejiang Energy Group Co., Ltd., Hangzhou 310007, P. R. China \\ * Corresponding author: Prof. Xiang GAO, Tel: +86-571-87951335, Fax: \\ +86-571-87951616, Email: xgao1@zju.edu.cn
}

Number of pages: 7

Number of Tables: 1

Number of Figures: 5

\section{List of Tables}

Table S1. Typical experimental conditions.

\section{List of Figures}

Figure S1. Flow field under different sampling positions with CFD study.

Figure S2. Effects of gas dilution on the size distribution of SAA.

Figure S3. Overall concentration change under different temperatures.

Figure S4. Visible droplet condensation on the tube surface.

Figure S5. Corona current and number concentration under different voltages. 
Table S1. Typical experimental conditions.

\begin{tabular}{cccccc}
\hline Items & $c_{\mathrm{SO}_{3}}$ & $\mathrm{~T}$ & $\tau$ & $\mathrm{w}$ & $\mathrm{U}$ \\
\hline Unit & $\mathrm{ppm}$ & ${ }^{\circ} \mathrm{C}$ & $\mathrm{s}$ & $\mathrm{g} / \mathrm{m}^{3}$ & $\mathrm{kV}$ \\
\hline Typical value & 10 & 45 & 5 & 0 & 0 \\
\hline Variation range & constant & $30 \sim 45$ & $3.7 \sim 27$ & $0 \sim 160$ & $0 \sim 12$ \\
\hline
\end{tabular}




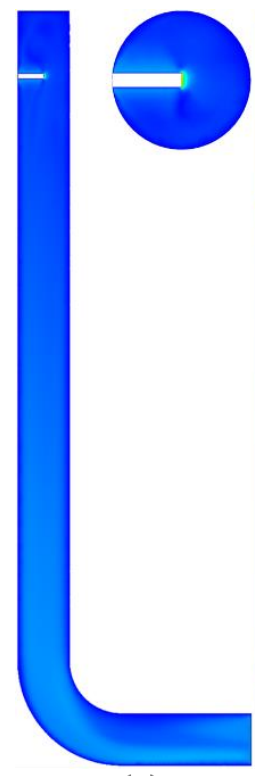

(a)

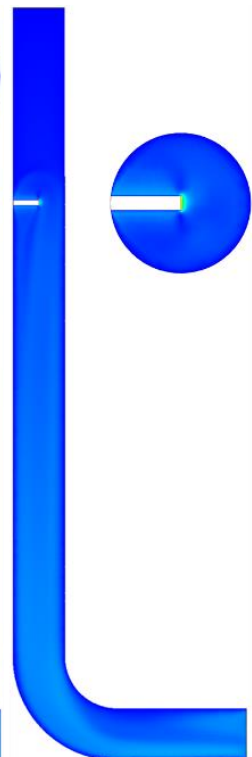

(b)

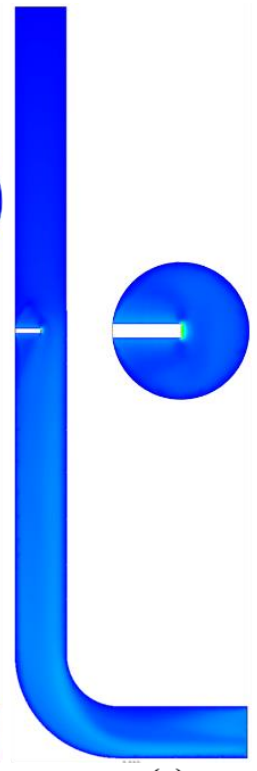

(c)

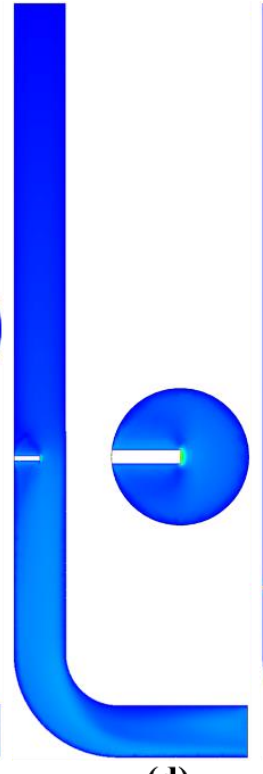

(d)

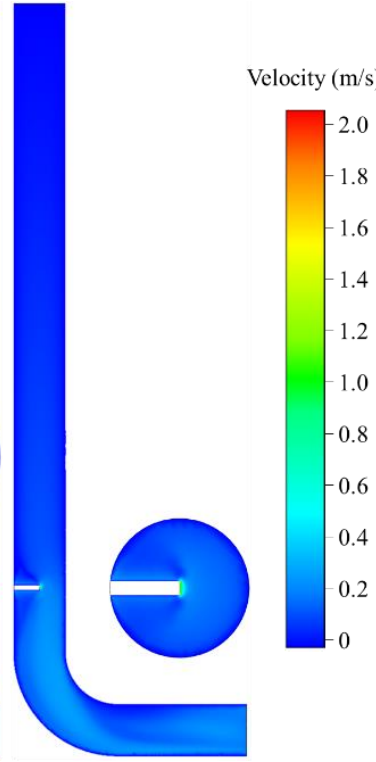

(e)

Figure S1. Flow field under different sampling positions with CFD study. 


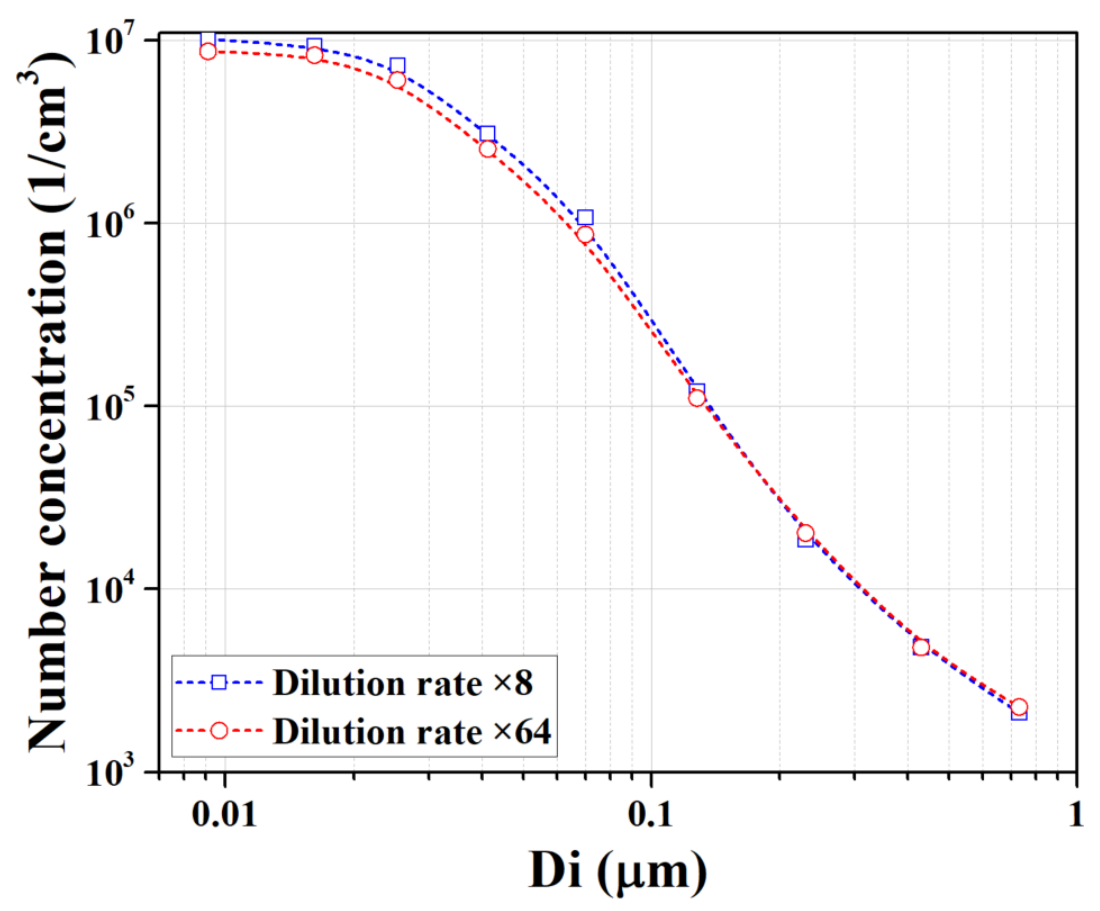

Figure S2. Effects of gas dilution on the size distribution of SAA. 

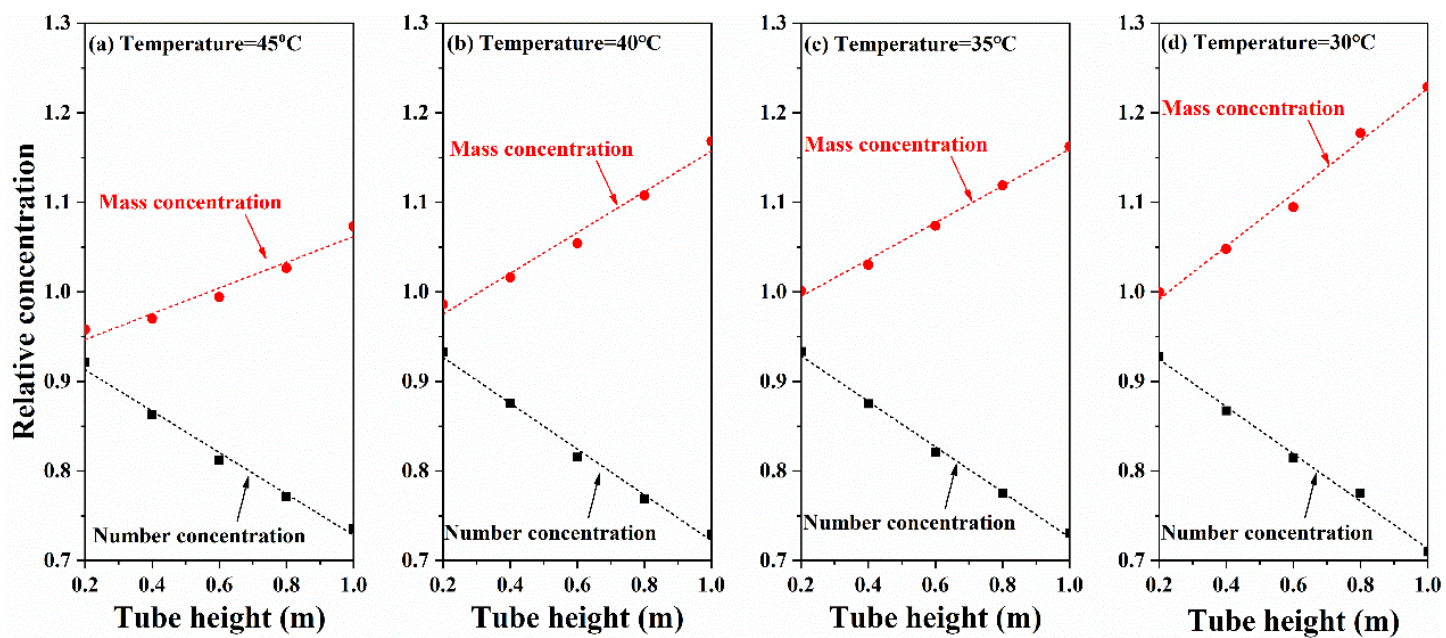

Figure S3. Overall concentration change under different temperatures. 


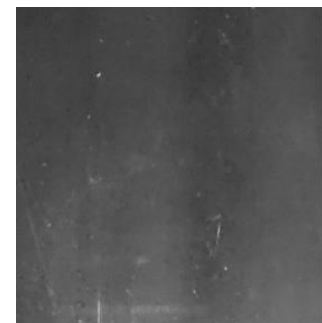

$45^{\circ} \mathrm{C}$

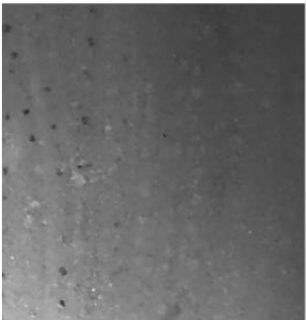

$40^{\circ} \mathrm{C}$

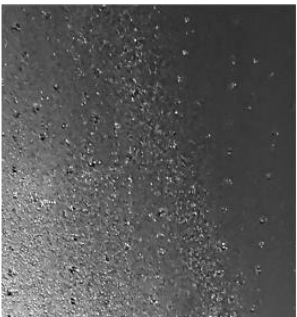

$35^{\circ} \mathrm{C}$

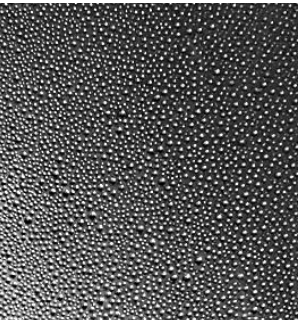

$30^{\circ} \mathrm{C}$

Figure S4. Visible condensation droplet on the tube surface. 


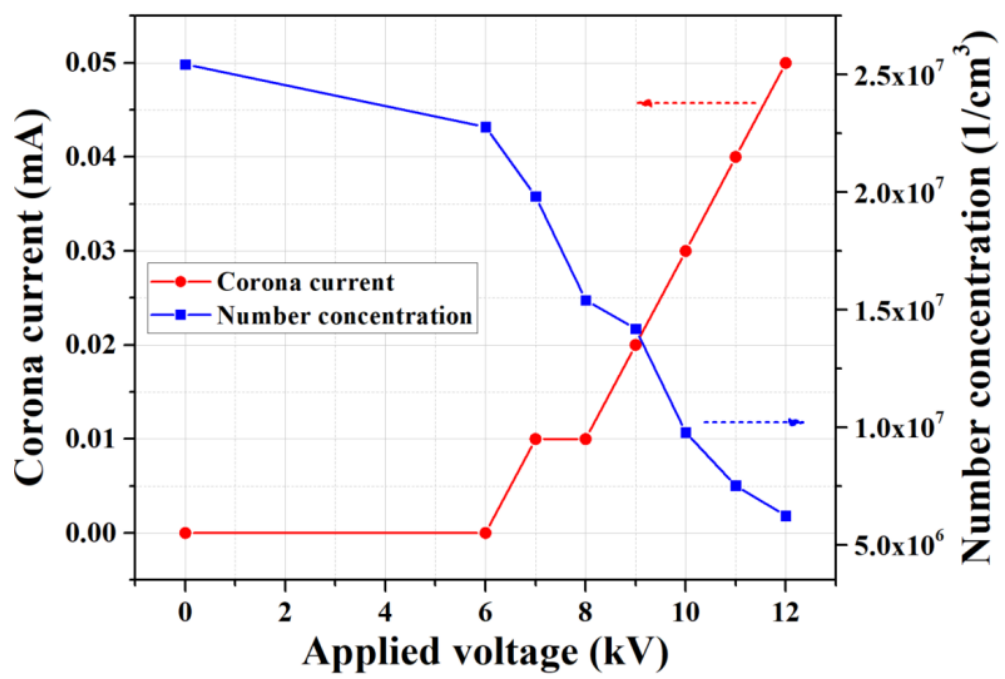

Figure S5. Corona current and number concentration under different voltages. 